Moroccan J. of Pure and Appl. Anal. (MJPAA)

Volume 7(1), 2021, Pages 01-11

ISSN: Online 2351-8227 - Print 2605-6364

DOI: $10.2478 / \mathrm{mjpaa}-2021-0001$

\title{
A new conjugate gradient method for acceleration of gradient descent algorithms
}

\author{
Noureddine Rahali ${ }^{1}$, Mohammed Belloufi $^{2}$, And Rachid Benzine ${ }^{3}$
}

AвSTRACT. An accelerated of the steepest descent method for solving unconstrained optimization problems is presented. which propose a fundamentally different conjugate gradient method, in which the well-known parameter $\beta_{k}$ is computed by an new formula. Under common assumptions, by using a modified Wolfe line search, descent property and global convergence results were established for the new method. Experimental results provide evidence that our proposed method is in general superior to the classical steepest descent method and has a potential to significantly enhance the computational efficiency and robustness of the training process.

Mathematics Subject Classification (2020). 65K05, 90C25, 90C26, 90C27, 90C30.

Key words and phrases. Unconstrained optimization, Conjugate gradient method, Line search, Global convergence.

Received : 05 July 2020 - Accepted: 12 October 2020.

(C)The Author(s) 2020. This article is published with open access by Sidi Mohamed Ben Abdallah University.

${ }^{1}$ University Ferhat Abbas Setif 1, Setif, 19000 and University Center of Tamanrasset, 11000, Algeria.

e-mail: rahalinour41@gmail.com (Corresponding Author).

${ }^{2}$ Laboratory Informatics and Mathematics (LiM), Mohamed Cherif Messaadia University, Souk Ahras, 41000, Algeria.

e-mail:m.belloufi@univ-soukahras.dz

${ }^{3}$ Superior School of Industrial Technologies Annaba, Annaba 23000, Algeria.

e-mail: rabenzine@yahoo.fr. 


\section{Introduction}

Consider the unconstrained optimization problem

$$
\left\{\min f(x), \quad x \in \mathbb{R}^{n}\right\},
$$

where $f: \mathbb{R}^{n} \longrightarrow \mathbb{R}$ is continuously differentiable. The line search method usually takes the following iterative formula

$$
x_{k+1}=x_{k}+\alpha_{k} d_{k}
$$

for (1.1), where $x_{k}$ is the current iterate point, $\alpha_{k}>0$ is a steplength and $d_{k}$ is a search direction. Different choices of $d_{k}$ and $\alpha_{k}$ will determine different line search methods [10, 18, 13]. We denote $f\left(x_{k}\right)$ by $f_{k}, \nabla f\left(x_{k}\right)$ by $g_{k}$, and $\nabla f\left(x_{k+1}\right)$ by $g_{k+1}$, respectively. $\|$.$\| denotes the$ Euclidian norm of vectors and define $y_{k}=g_{k+1}-g_{k}$.

We all know that a method is called steepest descent method if we take $d_{k}=-g_{k}$ as a search direction at every iteration, which has wide applications in solving large-scale minimization problems $[24,25,15]$. One drawback of the method is often yielding zigzag phenomena in solving practical problems, which makes the algorithm converge to an optimal solution very slowly, or even fail to converge [21,20].

If we take $d_{k}=-H_{k} g_{k}$ as a search direction at each iteration in the algorithm, where $H_{k}$ is an $n \times n$ matrix approximating $\left[\nabla^{2} f\left(x_{k}\right)\right]^{-1}$, then the corresponding method is called the Newton-like method [21, 20,27] such as the Newton method, the quasi-Newton method, variable metric method, etc. Many papers have proposed this method for optimization problems $[8,9,5]$.

However, the Newton-like method needs to store and compute matrix $H_{k}$ at each iteration and thus adds to the cost of storage and computation. Accordingly, this method is not suitable to solve large-scale optimization problems in many cases.

The steepest descent method is one of the simplest and the most fundamental minimization methods for unconstrained optimization. Since it uses the negative gradient as its descent direction, it is also called the gradient method.

For many problems, the steepest descent method is very slow. Although the method usually works well in the early steps, as a stationnary point is approached, it descends very slowly with zigzaguing phenomena. There are some ways to overcome these difficulties of zigzagging by defleting the gradient. Rather then moving along $d_{k}=-\nabla f\left(x_{k}\right)=-g_{k}$, we can move along

$$
d_{k}=-D_{k} \nabla f\left(x_{k}\right),
$$

or along

$$
d_{k}=-g_{k}+h_{k}
$$

where $D_{k}$ is an appropriate matrix and $h_{k}$ is an appropriate vector.

Due to its simplicity and its very low memory requirement, the conjugate gradient method is a powerful line search method for solving the large-scale optimization problems. In fact, the CG method is not among the fastest or most robust optimization algorithms for nonlinear problems available today, but it remains very popular for engineers and mathematicians who are interested in solving large problems $[12,3,11,6,28,26]$. The conjugate gradient method is designed to solve unconstrained optimization problem (1.1). More explicitly, the conjugate 
gradient method is an algorithm for finding the nearest local minimum of a function of variables which presupposes that the gradient of the function can be computed.We consider only the case where the method is implemented without regular restarts. The iterative formula of the conjugate gradient method is given by (1.2), where $\alpha_{k}$ is a steplength which is computed by carrying out a line search, and $d_{k}$ is the search direction defined by

$$
d_{k+1}= \begin{cases}-g_{k} & \text { si } k=1 \\ -g_{k+1}+\beta_{k} d_{k} & \text { si } k \geq 2\end{cases}
$$

where $\beta_{k}$ is a scalar, and $g_{k}$ denotes $g\left(x_{k}\right)$. Some well known formulas for $\beta_{k}$ are given as follows:

$$
\begin{aligned}
& \beta_{k}^{H S}=\frac{g_{k+1}^{T} y_{k}}{d_{k}^{T} y_{k}}, \beta_{k}^{F R}=\frac{\left\|g_{k+1}\right\|^{2}}{\left\|g_{k}\right\|^{2}}, \beta_{k}^{P R P}=\frac{g_{k+1}^{T} y_{k}}{\left\|g_{k}\right\|^{2}}, \beta_{k}^{C D}=-\frac{\left\|g_{k+1}\right\|^{2}}{d_{k}^{T} g_{k}} \\
& \beta_{k}^{L S}=-\frac{g_{k+1}^{T} y_{k}}{d_{k}^{T} g_{k}}, \beta_{k}^{D Y}=\frac{\left\|g_{k+1}\right\|^{2}}{d_{k}^{T} y_{k}}, \beta_{k}^{H Z}=\left(y_{k}-2 d_{k} \frac{\left\|y_{k}\right\|^{2}}{d_{k}^{T} y_{k}}\right)^{T} \frac{g_{k+1}}{d_{k}^{T} y_{k}}
\end{aligned}
$$

The above corresponding methods are known as Hestenes-Stiefel (HS) method [16], the Fletcher-Reeves (FR) method [14], the Polak-Ribière-Polyak (PR) method (see [23, 4]), the Conjugate Descent method(CD) [14], the Liu-Storey (LS) method [19], the Dai-Yuan (DY) method [12], and Hager and Zhang (HZ) method [17], respectively.

In the convergence analysis and implementation of conjugate gradient methods, one often requires the inexact line search such as theWolfe conditions or the strong Wolfe conditions. The Wolfe line search is to find $\alpha_{k}$ such that

$$
\begin{gathered}
f\left(x_{k}+\alpha_{k} d_{k}\right) \leq f\left(x_{k}\right)+\delta \alpha_{k} g_{k}^{T} d_{k} \\
d_{k}^{T} g\left(x_{k}+\alpha_{k} d_{k}\right) \geq \sigma d_{k}^{T} g_{k},
\end{gathered}
$$

with $\delta<\sigma<1$. The strong Wolfe line search is to find $\alpha_{k}$ such that

$$
\begin{gathered}
f\left(x_{k}+\alpha_{k} d_{k}\right) \leq f\left(x_{k}\right)+\delta \alpha_{k} g_{k}^{T} d_{k} \\
\left|d_{k}^{T} g\left(x_{k}+\alpha_{k} d_{k}\right)\right| \leq-\sigma d_{k}^{T} g_{k},
\end{gathered}
$$

where $\delta<\sigma<1$ are constants.

The convergence behavior of the above formulas with some line search conditions has been studied by many authors for many years.

Al-Baali [1] has proved the global convergence of the FR method for nonconvex functions with the strongWolfe line search if the parameter $\sigma<\frac{1}{2}$. The PRP method with exact line search may cycle without approaching any stationary point, see Powell's counter-example [22]. Although one would be satisfied with its global convergence properties, the FR method sometimes performs much worse than the PRP method in real computations. A similar case happen to the DY method and the HS method.

In next section, we will state the idea of the new method, then a new algorithm will be developed. Descent property and the global convergence will be established in Section 3 . Section 4 is devoted to numerical experiments by implementing the algorithm to solve many large-scale benchmark test problems. The conclusions are presented in Section 5. 


\section{The new formula and the corresponding algorithm}

In this section, we shall state the idea to propose a new conjugate gradient method and develop a new algorithm.

In this paper, based the modified strong Wolfe type line search, under some mild conditions, we give the Descent property and global convergence of the new $\beta_{k}$ which is known as $\beta_{k}^{B R B}$, where $B R B$ denotes Belloufi, Rahali and Benzine. Then we can define the following formulas $\beta_{k}$ to compute the search directions in (1.4).

$$
\beta_{k}^{B R B}=\frac{\left\|g_{k+1}\right\|^{2}}{\left\|d_{k}\right\|^{2}}
$$

With the constructed search direction, we find a stepsize by the modified strong Wolfe line search strategy:

\section{Modification of the strong Wolfe line search}

The step length is computed by performing a line search along $d_{k}$. In practice, a relevant choice is to compute $\alpha_{k}$ according to the realization of the modified strong Wolfe conditions, namely

$$
\begin{gathered}
f\left(x_{k}+\alpha_{k} d_{k}\right)-f\left(x_{k}\right) \leq \delta \alpha_{k} g_{k}^{T} d_{k} \\
\left|g\left(x_{k}+\alpha_{k} d_{k}\right)^{T} d_{k}\right| \leq-\sigma g_{k}^{T} d_{k} \frac{\left\|d_{k}\right\|^{2}}{\left\|g_{k}\right\|^{2}}
\end{gathered}
$$

The algorithm is given as follows:

algorithm Step 0: Given $x_{1} \in \mathbb{R}^{n}$, set $d_{1}=-g_{1}, k:=1$.

Step 1: If $\left\|g_{k}\right\|=0$ then stop else go to Step 2 .

Step 2: Set $x_{k+1}=x_{k}+a_{k} d_{k}$ where $d_{k}$ is defined by (1.4),(2.1) and $\alpha_{k}$ is defined by (2.2),(2.3). Step 3: Set $k:=k+1$ and go to Step 1 .

\section{Descent property and global convergence}

The following theorem indicates that, in the inexact case, the search direction $d_{k}$ satisfies descent property.

Theorem 3.1. If an $\alpha_{k}$ is calculated wich satisfies modified strong Wolfe line search (2.2) and (2.3) with $\left.\sigma \in] 0, \frac{1}{2}\right], \forall k$ then for the new conjugate gradient method, the inequality

$$
-\sum_{j=0}^{k-1} \sigma^{j} \leq \frac{g_{k}^{T} d_{k}}{\left\|g_{k}\right\|^{2}} \leq-2+\sum_{j=0}^{k-1} \sigma^{j}
$$

holds for all $k$, and hence the descent property

$$
g_{k}^{T} d_{k}<0, \forall k
$$

holds, as long as $g_{k} \neq 0$. 
Proof. The proof is by induction. For $k=1$ Equations (3.1) and (3.2) is clearly satisfied.

Now we suppose that (3.1) and (3.2) hold for any $k \geq 1$.

It follows from the definition (1.4) and (2.1) of $d_{k+1}$ that

$$
\frac{g_{k+1}^{T} d_{k+1}}{\left\|g_{k+1}\right\|^{2}}=-1+\frac{g_{k+1}^{T} d_{k}}{\left\|d_{k}\right\|^{2}}
$$

and hence from (2.3) and (3.2) that

$$
-1+\sigma \frac{g_{k}^{T} d_{k}}{\left\|g_{k}\right\|^{2}} \leq \frac{g_{k+1}^{T} d_{k+1}}{\left\|g_{k+1}\right\|^{2}} \leq-1-\sigma \frac{g_{k}^{T} d_{k}}{\left\|g_{k}\right\|^{2}}
$$

Also, by induction assumption (3.1), we have

$$
\begin{gathered}
-\sum_{j=0}^{k} \sigma^{j}=-1-\sigma \sum_{j=0}^{k-1} \sigma^{j} \leq \frac{g_{k+1}^{T} d_{k+1}}{\left\|g_{k+1}\right\|^{2}} \\
\leq-1+\sigma \sum_{j=0}^{k-1} \sigma^{j}=-2+\sum_{j=0}^{k} \sigma^{j}
\end{gathered}
$$

Then, (3.1) holds for $k+1$.

Since

$$
g_{k+1}^{T} d_{k+1} \leq\left\|g_{k+1}\right\|^{2}\left(-2+\sum_{j=0}^{k} \sigma^{j}\right)
$$

and

$$
\sum_{j=0}^{k} \sigma^{j}<\sum_{j=0}^{\infty} \sigma^{j}=\frac{1}{1-\sigma}
$$

where $\left.\sigma \in] 0, \frac{1}{2}\right]$, it follows from $1-\sigma>\frac{1}{2}$ that $-2+\sum_{j=0}^{k} \sigma^{j}<0$. Hence, from (3.5), we obtain $g_{k+1}^{T} d_{k+1}<0$. We complete the proof by induction.

In order to establish the global convergence of the proposed method, we assume that the following assumption always holds, i.e. Assumption 3.1 :

Assumption 3.1:

Let $f$ be twice continuously differentiable, and the level set $L=\left\{x \in \mathbb{R}^{n} \mid f(x) \leq f\left(x_{1}\right)\right\}$ be bounded

Theorem 3.2. Suppose that $x_{1}$ is a starting point for which Assumption 3.1 holds. Consider the New method (1.4) and (2.1). If the steplength $\alpha_{k}$ is computed by the modified strong Wolfe line search (2.2) and (2.3) with $\delta<\sigma<\frac{1}{2}$ and if

$$
\frac{1}{\left\|d_{k-1}\right\|^{4}} \leq \frac{1}{\left\|g_{k-1}\right\|^{4}}
$$

then the method is globally convergent, i.e.,

$$
\liminf _{k \rightarrow \infty}\left\|g_{k}\right\|=0
$$


Proof. It is shown in theorem 1 that the descent property (3.2) holds for $\left.\sigma \in] 0, \frac{1}{2}\right]$, so from (2.3), (3.1), and (3.6) it follows that

$$
\left|g_{k}^{T} d_{k-1}\right| \leq-\sigma g_{k-1}^{T} d_{k-1} \frac{\left\|d_{k-1}\right\|^{2}}{\left\|g_{k-1}\right\|^{2}} \leq\left\|d_{k-1}\right\|^{2} \sigma \sum_{j=0}^{k-2} \sigma^{j}=\left\|d_{k-1}\right\|^{2} \sum_{j=0}^{k-1} \sigma^{j} \leq \frac{\sigma}{1-\sigma}\left\|d_{k-1}\right\|^{2}
$$

Thus from the definition of $d_{k}$ and using (2.1) and (3.9) we deduce that

$$
\begin{gathered}
\left\|d_{k}\right\|^{2}=\left\|g_{k}\right\|^{2}-2 \beta_{k-1} g_{k}^{T} d_{k-1}+\beta_{k-1}^{2}\left\|d_{k-1}\right\|^{2} \\
\leq\left\|g_{k}\right\|^{2}+\frac{2 \sigma}{1-\sigma} \beta_{k}\left\|d_{k-1}\right\|^{2}+\beta_{k-1}^{2}\left\|d_{k-1}\right\|^{2} \\
=\left(\frac{1+\sigma}{1-\sigma}\right)\left\|g_{k}\right\|^{2}+\frac{\left\|g_{k}\right\|^{4}}{\left\|d_{k-1}\right\|^{4}}\left\|d_{k-1}\right\|^{2} \\
\leq\left(\frac{1+\sigma}{1-\sigma}\right)\left\|g_{k}\right\|^{2}+\frac{\left\|g_{k}\right\|^{4}}{\left\|g_{k-1}\right\|^{4}}\left\|d_{k-1}\right\|^{2}
\end{gathered}
$$

where we used the facts that

$$
\frac{1}{\left\|d_{k-1}\right\|^{4}} \leq \frac{1}{\left\|g_{k-1}\right\|^{4}}
$$

By applying this relation repeatedly, it follows that

$$
\left\|d_{k}\right\|^{2} \leq\left(\frac{1+\sigma}{1-\sigma}\right)\left\|g_{k}\right\|^{4} \sum_{j=1}^{k} \frac{1}{\left\|g_{j}\right\|^{2}}
$$

Now we prove (3.8) by contradiction. It assumes that (3.8) does not hold, then there exists a constant $\varepsilon>0$ such that

$$
\left\|g_{k}\right\| \geq \varepsilon>0
$$

holds for all $k$ sufficiently large. Since $g_{k}$ is bounded above on the level set $L$, it follows from (3.11) that

$$
\left\|d_{k}\right\|^{2} \leq c_{1} k
$$

where $c_{1}$ is a positive constant. From (3.1) and (3.6), we have

$$
\cos \theta_{k} \geq\left(\frac{1-2 \sigma}{1-\sigma}\right) \frac{\left\|g_{k}\right\|}{\left\|d_{k}\right\|}
$$

Since $\sigma<\frac{1}{2}$, substituting (3.13) and (3.12) into (3.14) gives

$$
\sum_{k} \cos ^{2} \theta_{k} \geq\left(\frac{1-2 \sigma}{1-\sigma}\right)^{2} \sum_{k} \frac{\left\|g_{k}\right\|^{2}}{\left\|d_{k}\right\|^{2}} \geq c_{2} \sum_{k} \frac{1}{k}
$$

where $c_{2}$ is a positive constant. Therefore, the series $\sum_{k} \cos ^{2} \theta_{k}$ is divergent.

Let $M$ be an upper bound of $\left\|\nabla^{2} f(x)\right\|$ on the level set $L$, then

$$
g_{k+1}^{T} d_{k}=\left(g_{k}+a_{k} \nabla^{2} f(x)\right)^{T} d_{k} \leq g_{k}^{T} d_{k}+M a_{k}\left\|d_{k}\right\|^{2}
$$


Thus by using (2.3) and (3.7) we obtain

$$
a_{k} \geq-\frac{(1-\sigma)}{M\left\|d_{k}\right\|^{2}} g_{k}^{T} d_{k}
$$

Substituting $a_{k}$ of (3.16) into (2.2) gives

$$
f_{k+1} \leq f_{k}-c_{3}\left\|g_{k}\right\|^{2} \cos ^{2} \theta_{k}
$$

where $c_{3}=\frac{(1-\sigma) \delta}{M}>0$. Since $f(x)$ is bounded below, $\sum_{k}\left\|g_{k}\right\|^{2} \cos ^{2} \theta_{k}$ converges, which indicates that $\sum_{k} \cos ^{2} \theta_{k}$ converges by use of (3.12). This fact contradicts (3.15). We complete the proof.

\section{Numerical results and discussions}

In this section we report some numerical results obtained with a Fortran implementation of gradient algorithms and their accelerated variants. All codes are written in Fortran and compiled with f77 (default compiler settings) on a Workstation Intel(R) core(TM), i3@ 2.20GHz. We selected a number of 75 large-scale unconstrained optimization test functions in generalized or extended form [2]. For each test function we have considered ten numerical experiments with the number of variables $n=1000,2000, \ldots, 10000$. In the following we present the numerical performance of CG and ACG codes corresponding to different formula for $\beta_{k}$ computation. All algorithms implement the Wolfe line search conditions with $\rho=0.0001$ andr $\sigma=0.9$, and the same stopping criterion $\left\|g_{k}\right\|_{\infty} \leq 10^{-10}$, where $\|\cdot\|_{\infty}$ is the maximum absolute component of a vector.

The comparisons of algorithms are given in the following context. Let $f_{i}^{A L G 1}$ and $f_{i}^{A L G 2}$ be the optimal value found by ALG1 and ALG2, for problem $i=1, \ldots, 750$, respectively. We say that, in the particular problem $i$, the performance of ALG1 was better than the performance of ALG2 if:

$$
\left|f_{i}^{A L G 1}-f_{i}^{A L G 2}\right|<10^{-3},
$$

and the number of iterations, or the number of function-gradient evaluations, or the CPU time of ALG1 was less than the number of iterations, or the number of function-gradient evaluations, or the CPU time corresponding to ALG2, respectively.

We compare the New method CGBRB with the steepest descent method, the CG_DESCENT method, and PRP conjugate gradient method.

Figures 1-4 list the performance of the CGBRB, steepest descent, CG_DESCNET and PRP conjugate gradient methods. Relative to CPU time, the number of iterations and the number of gradient evaluations, respectively, which were evaluated using the profiles of Dolan and Moré [7].

Clearly, Figures 1-4 present that our proposed method CGBRB exhibits the best overall performance since it illustrates the highest probability of being the optimal solver, followed by the steepest descent, CG_DESCNET, and PRP conjugate gradient methods relative to all performance metrics. 


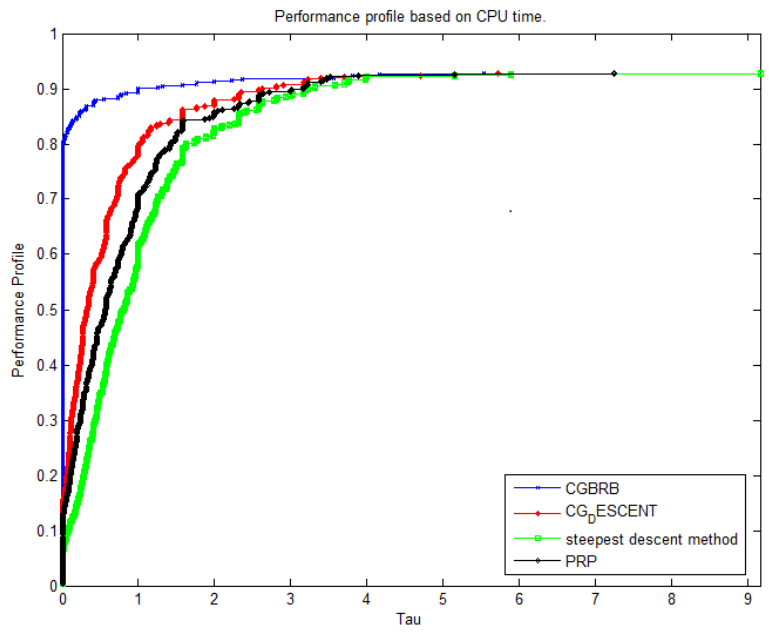

Figure 1. Performance based on CPU time.

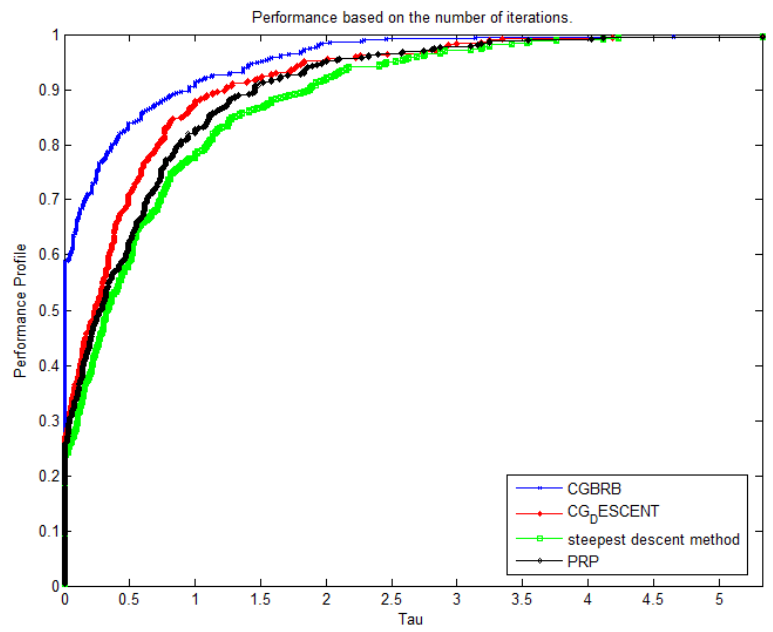

FIGURE 2. Performance based on the number of iterations. 


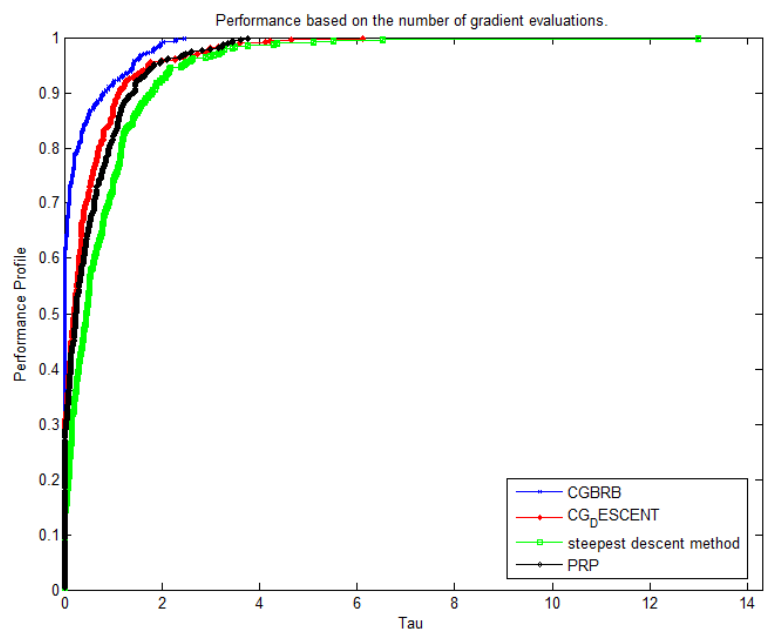

Figure 3. Performance based on the number of function evaluations.

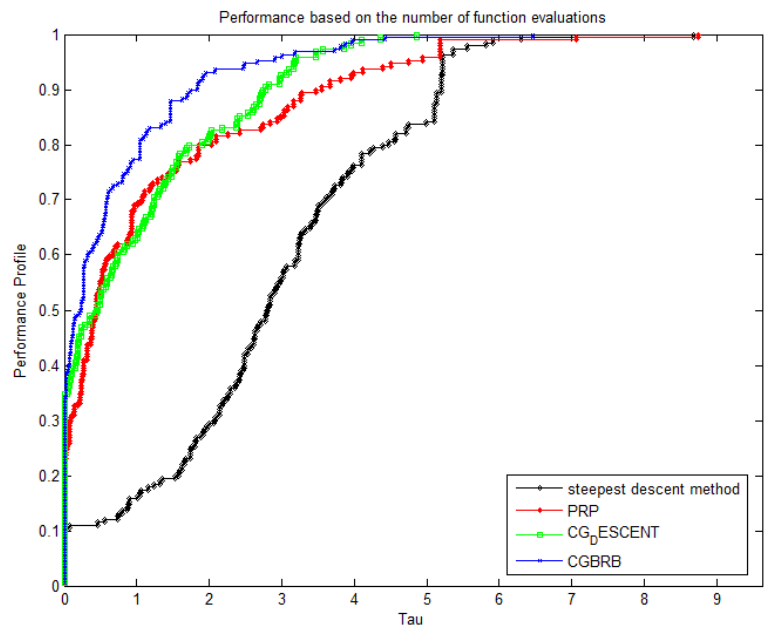

Figure 4. Performance based on the number of gradient evaluations. 


\section{Conclusion}

We have presented a new conjugate gradient algorithm for solving unconstrained optimization problems. Under the modified strong Wolfe line search conditions we proved the descent property and global convergence of the algorithm. For the test problems, the comparison of the numerical results shows that the new algorithms is a good search direction at every iteration.

\section{References}

[1] M. Al-Baali, Descent property and global convergence of the Fletcher-Reeves method with inexact line search, IMA J. Numer. Anal. 5 (1985) 121-124.

[2] N. Andrei, An unconstrained optimization test functions collection, Advanced Modeling and Optimization, An Electronic International Journal 10 (2008) 147-161.

[3] M. Belloufi, R. Benzine, Descent property and global convergence of a new search direction method for unconstrained optimization, Numerical Functional Analysis and Optimization , 36(2), 169-180, Taylor \& Francis Group 2014.

[4] Sh. Sadigh Behzadi and A. Yildirim, Numerical solution of lr fuzzy hunter-saxeton equation by using homotopy analysis method, Journal of Applied Analysis and Computation, 2012, Vol.2, (1):1-10.

[5] R. Benouahboun, A. Mansouri, An interior point algorithm for convex quadratic programming with strict equilibrium. RAIRO Oper. Res. 39 (2005) 13-33.

[6] M.Q. Chen, S. -P. Han, A parallel quasi-Newton method for partially separable large scale minimization, Annals of Operations Research, 1988, Volume 14, Issue 1 , pp 195-211.

[7] E. Dolan, J. J. Moré (2002). Benchmarking optimization software with performance profiles. Mathematical Programming 91:201-213.

[8] Y. Dai, Convergence properties of the BFGS algorithm. SIAM Journal on Optimization, 13 (2003) 693-701.

[9] J. E. Dennis, Jr., J. J. Moré, (1977). Quasi-Newton methods, motivation and theory. SIAM Review, 19, 46-89.

[10] X. Dong, W.j. Li, Y.b. He, Some modified Yabe-Takano Conjugate gradient methods with Sufficient descent condition. RAIRO-Oper. Res. 2016.DOI: http://dx.doi.org/10.1051/ro/2016028.

[11] Sarra Delladji, Mohammed Belloufi, Badereddine Sellami, Behavior of the combination of PRP and HZ methods for unconstrained optimization, Numerical Algebra Control And Optimization, (2020), doi:10.3934/naco.2020032

[12] Y.H. Dai, Y. Yuan, An Efficient Hybrid Conjugate Gradient Method for Unconstrained Optimization, Annals of Operations Research, March 2001, Volume 103, Issue 1-4 , pp 33-47

[13] S. Deng, Coercivity properties and well-posedness in vector optimization. RAIRO Oper. Res. 37 (2003) 195208.

[14] R. Fletcher, Practical Method of Optimization, second ed., Unconstrained Optimization, vol. I, Wiley, New York, 1997.

[15] M.C. Ferris, A.J. Wathen, P. Armand, Limited memory solution of bound constrained convex quadratic problems arising in video games. RAIRO Oper. Res. 41 (2007) 19-34.

[16] M.R. Hestenes, E. Stiefel, Method of conjugate gradient for solving linear equations, J. Res. Nat. Bur. Stand. 49 (1952) 409-436.

[17] W.W. Hager, H. Zhang, A new conjugate gradient method with guaranteed descent and an efficient line search, SIAM Journal on Optimization 16 (2005) 170-192.

[18] S.B. Kafaki, R. Ghanbari, A descent hybrid modification of the Polak-Ribière-Polyak conjugate gradient method. RAIRO-Oper. Res. 50 (2016) 567-574.

[19] Y. Liu, C. Storey, Efficient generalized conjugate gradient algorithms. Part 1: Theory, J. Optimiz. Theory Appl. 69 (1992) 129-137. 
[20] J. Nocedal, S.J. Wright, Numerical optimization, Springer Series in Operations Research, Springer, New York, 1999.

[21] J. Nocedal, Conjugate gradient methods and nonlinear optimization, in: L. Adams, J.L. Nazareth (Eds.), Linear and Nonlinear Conjugate Gradient Related Methods, SIAM, Philadelphia, PA, 1995, pp. 9-23.

[22] M.J.D. Powell, Nonconvex minimization calculations and the conjugate gradient method, Lecture Notes in Math. 1066 (1984) 121-141.

[23] B.T. Polyak, The conjugate gradient method in extreme problems, USSR Comp. Math. Math. Phys. 9 (1969) 94-112.

[24] M. A. Rincon, M. I. M. Copetti, Numerical analysis for a locally damped wave equation, Journal of Applied Analysis and Computation, 2013, Vol.3, (2):169-182.

[25] J. Schropp, A note on minimization problems and multistep methods. Numeric Mathematic, 78 (1997) 87101.

[26] N.H. Zhao, Y. Xia, W. Liu, P. J. Y. Wong and R. T. Wang, Existence of Almost Periodic Solutions of a Nonlinear System, Journal of Applied Analysis and Computation, 2013, Vol.3, (3):301-306.

[27] Z.J. Shi, X.S. Zhang, J. Shen, Convergence analysis of adaptive trust region methods. RAIRO Oper. Res. 41 (2007) 105-121.

[28] C. Xu, J. Zhang, A Survey of Quasi-Newton Equations and Quasi-Newton Methods for Optimization, Annals of Operations Research,2001, Volume 103, Issue 1-4 , pp 213-234. 